\title{
Carcinoma Exhibiting Undifferentiated or Dedifferentiated Features
}

National Cancer Institute

\section{Source}

National Cancer Institute. Carcinoma Exhibiting Undifferentiated or Dedifferentiated

Features. NCI Thesaurus. Code C159465.

A microscopic finding that refers to a carcinoma which is composed either exclusively of an undifferentiated cellular infiltrate, or a mixture of well differentiated and undifferentiated cellular infiltrates. 